\title{
Spectrophotometric and fluorometric methods for the determination of Fe(III) ions in water and pharmaceutical samples
}

\author{
Ahmed Shahat ${ }^{1, *}$, Nuha Y. Elamin ${ }^{2,3}$, Wesam Abd El-Fattah ${ }^{2,4}$ \\ ${ }^{1}$ Chemistry Department, Faculty of Science, Suez University, Suez 43518, Egypt \\ ${ }^{2}$ Chemistry Department, College of Science, IMSIU (Imam Mohammad Ibn Saud Islamic \\ University), Riyadh 11623, kingdom of Saudi Arabia \\ ${ }^{3}$ Department of Chemistry, Sudan University of Science and Technology, P.O. Box 407, \\ Khartoum, Sudan \\ 4 Department of Chemistry, Faculty of Science, Port-Said University, Port-Said, Egypt
}

*Corresponding author E-mail: ashahat@aucegypt.edu (Ahmed Shahat) 


\section{Synthesis of the mesoporous silica nanotubes (MSNTs)}

Mesoporous silica nanotubes have been synthesized using the same steps for preparing the silica nanotubes $^{1}$ but with some modifications. Typically, 4.5 g Brij C10 was dissolved in $10 \mathrm{~mL}$ cyclohexane and reserved at $50{ }^{\circ} \mathrm{C} .1 .3 \mathrm{~mL}$ solution of $\mathrm{NiCl}_{2}(0.8 \mathrm{M})$ was added under agitation. Subsequently, $0.45 \mathrm{~mL}$ hydrazine hydrate was added to form nanorods from the nickel hydrazine complex. The suspended solution was lifted under stirring for $3 \mathrm{~h}$. Then, $1 \mathrm{~mL}$ diethylamine was added and the reaction mixture was lifted to stirring for more $1.5 \mathrm{~h}$. At that time, $10 \mathrm{~mL}$ ethanol containing 3.0 gm cetyltrimethylammonium bromide (CTAB: Sigma-Aldrich, USA) was added and the reaction mixture was stirred for $30 \mathrm{~min}$ (Scheme S1). Then, $3.0 \mathrm{~mL}$ tetraethyl orthosilicate (TEOS) (www.sigmaaldrich.com) was added for making the silica covering. The reaction mixture was allowed to stir for $3 \mathrm{~h}$ extra. Centrifugation was utilized for collecting the nickel hydrazine nanorod@silica core/shell material. Isopropanol was utilized for washing and storing the nanorod@silica core/shell precipitate. Acid etching was utilized for removing the nanorod core from inside the silica shell. Typically, the nickel hydrazine nanorod@silica core/shell was dispersed in $\mathrm{HCl}(35 \mathrm{~mL}, 1 \mathrm{M})$, and lifted under stirring for $1 \mathrm{~h}$. The MSNTs were collected by centrifugation, washed with deionized water, and dried at $80{ }^{\circ} \mathrm{C}$ for $24 \mathrm{~h}$. After that, they were calcined at $550^{\circ} \mathrm{C}$ for $8 \mathrm{~h}$ (Scheme S1).

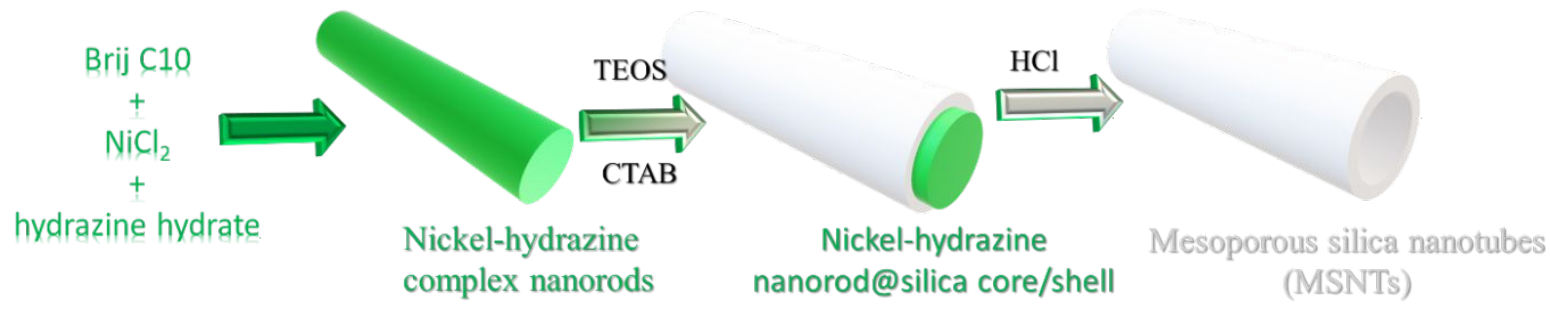


Scheme S1 The preparation of mesoporous silica nanotubes is depicted in a schematic diagram (MSNTs).

\section{The reaction between the 3-fsa-MSNTs and 5-fsa-MSNTs sensors with the Fe(III) ions}

UV-Vis spectrophotometric method (Shimadzu $2600 \mathrm{UV} / \mathrm{Vis}$ spectrophotometer, Made in Japan) was utilized for analyzing the multivariate between the 3-fsa-MSNTs and 5-fsa-MSNTs sensors with Fe(III) ions in the solution. These multivariate include $\mathrm{pH}$ effect, 3-fsa-MSNTs or 5fsa-MSNTs sensor repeatability, amount of sensor effect, interference studies, response time, reproducibility, stability, and dynamic linear concentration range.

The $\mathrm{pH}$ meter (JENWAY-370, England) was utilized for measuring the $\mathrm{pH}$. The $\mathrm{pH}$ 1-6 was prepared by using a mixture of $\left(\mathrm{KCl}-\mathrm{HCl}(0.2 \mathrm{M})\right.$ and $\mathrm{CH}_{3} \mathrm{COOH}-\mathrm{CH}_{3} \mathrm{COONa}(0.1 \mathrm{M})$. Also, 4-(2-hydroxyethyl)-1-piperazineethanesulfonic acid (HEPES) (0.1 M) with $\mathrm{NaOH}(0.1 \mathrm{M})$ were utilized for making the $\mathrm{pH}$ 7-9. The reaction between 3-fsa-MSNTs or 5-fsa-MSNTs sensor with $\mathrm{Fe}(\mathrm{III})$ ions at different $\mathrm{pHs}$ was studied from $\mathrm{pH} 1$ to $\mathrm{pH}$ 9. The amount of solid sensor study was carried by changing the amount of 3-fsa-MSNTs or 5-fsa-MSNTs sensor between 10 and $70 \mathrm{mg}$. The appropriate amount of sensor was added for reaction with $0.1 \mathrm{ppm}$ Fe(III) ions at the optimum pH 2.0, 4.0 for 3-fsa-MSNTs and 5-fsa-MSNTs sensor, respectively. Under the optimum reaction conditions, a response time study was conducted using Fe(III) ion concentration $0.1 \mathrm{ppm}$, and the absorption was taken every $30 \mathrm{~s}$ for $5 \mathrm{~min}$.

At the optimum $\mathrm{pH}$, the dynamic linear concentration range of $\mathrm{Fe}(\mathrm{III})$ ions with each sensor was evaluated. To a $10 \mathrm{~mL}$ buffered solution containing $20 \mathrm{mg}$ of 3-fsa-MSNTs and $30 \mathrm{mg}$ of 5fsa-MSNTs sensor, an appropriate amount of Fe(III) ions was added to change the concentration from $0.0 \mathrm{ppm}$ to $2.0 \mathrm{ppm}$. After stirring for $60 \mathrm{~s}$, the spectrum of the suspended solution was collected after each addition. Reproducibility analysis was studied by replicating each experiment 
three times. To examine the photodecomposition, the stability of the solid sensors study was performed by continuously exposing the suspended solutions of the 3-fsa-MSNTs or 5-fsa-MSNTs sensor at the optimum conditions to a light source (Xenon lamp) for $8 \mathrm{~h}$. An interference analysis using various foreign ions (Fe(II), $\mathrm{Mg}(\mathrm{II}), \mathrm{Ca}(\mathrm{II}), \mathrm{Ba}(\mathrm{II}), \mathrm{Co}(\mathrm{II}), \mathrm{Ni}(\mathrm{II}), \mathrm{Cu}(\mathrm{II}), \mathrm{Zn}(\mathrm{II}), \mathrm{Pb}(\mathrm{II})$, $\mathrm{Hg}(\mathrm{II})$, and $\mathrm{Cd}(\mathrm{II}))$ was conducted by adding various concentrations of these interfering ions to a solution containing $0.2 \mathrm{ppm}$ Fe(III) ion concentration, at the optimum conditions of each sensor. Then the absorption signal of 3-fsa-MSNTs or 5-fsa-MSNTs complexes was observed.

\section{Image processing}

For image processing, a light control box $(25 \mathrm{~cm} \times 15 \mathrm{~cm} \times 10 \mathrm{~cm})$ made of white foam board was used for the colorimetric detection of the Fe(III) ions. White foam was selected to help record color differences in photographs taken with the smartphone, as well as to monitor the luminosity and minimize external radiation interference. ${ }^{2}$ The digital images were taken with a Samsung Galaxy A71 mobile which has a rear camera (64-megapixel) and 10 operating system. It was held at a set distance $(7 \mathrm{~cm})$ from the samples in a holder on the box's side. The sample solutions of $\mathrm{Fe}(\mathrm{III})$ standard were added into a $10 \mathrm{~mL}$ colorless glass bottle containing $20 \mathrm{mg}$ of 3-fsa-MSNTs or $30 \mathrm{mg}$ of 5-fsa-MSNTs sensors suspended under their optimum conditions. By using our eyes, we could see the color change of the sensors in less than $1 \mathrm{~min}$. The smartphone's built-in camera was then used to take digital images. Every image's RGB values were measured using the Just Color Picker Software V 5.5, which is available for free on the internet, and used a computer for further analysis of the mean color intensity. 


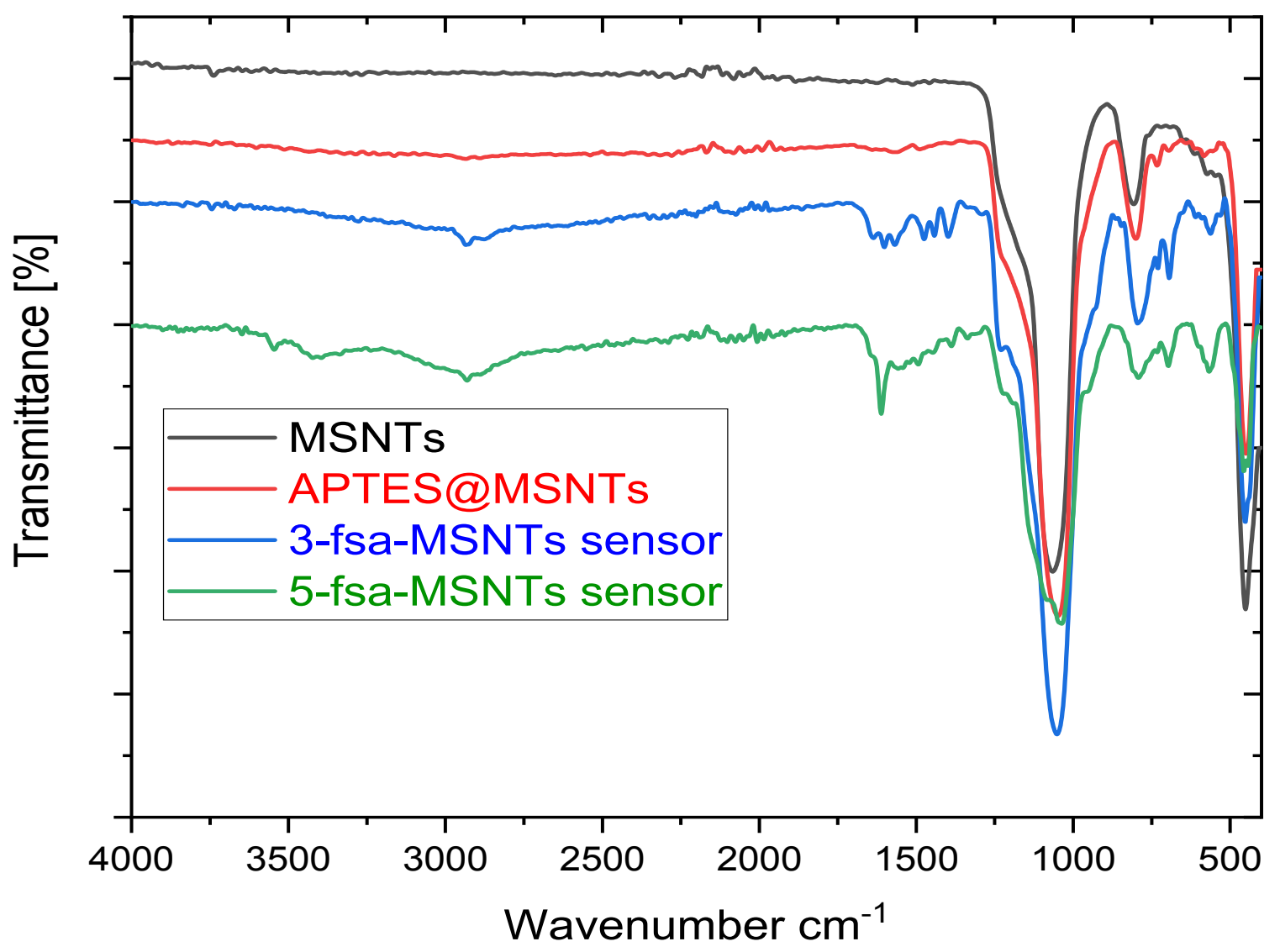

Figure S1. FTIR spectra of the MSNTs, 3-APTES@MSNTs, 3-fsa-MSNTs and 5-fsa-MSNTs sensors. 

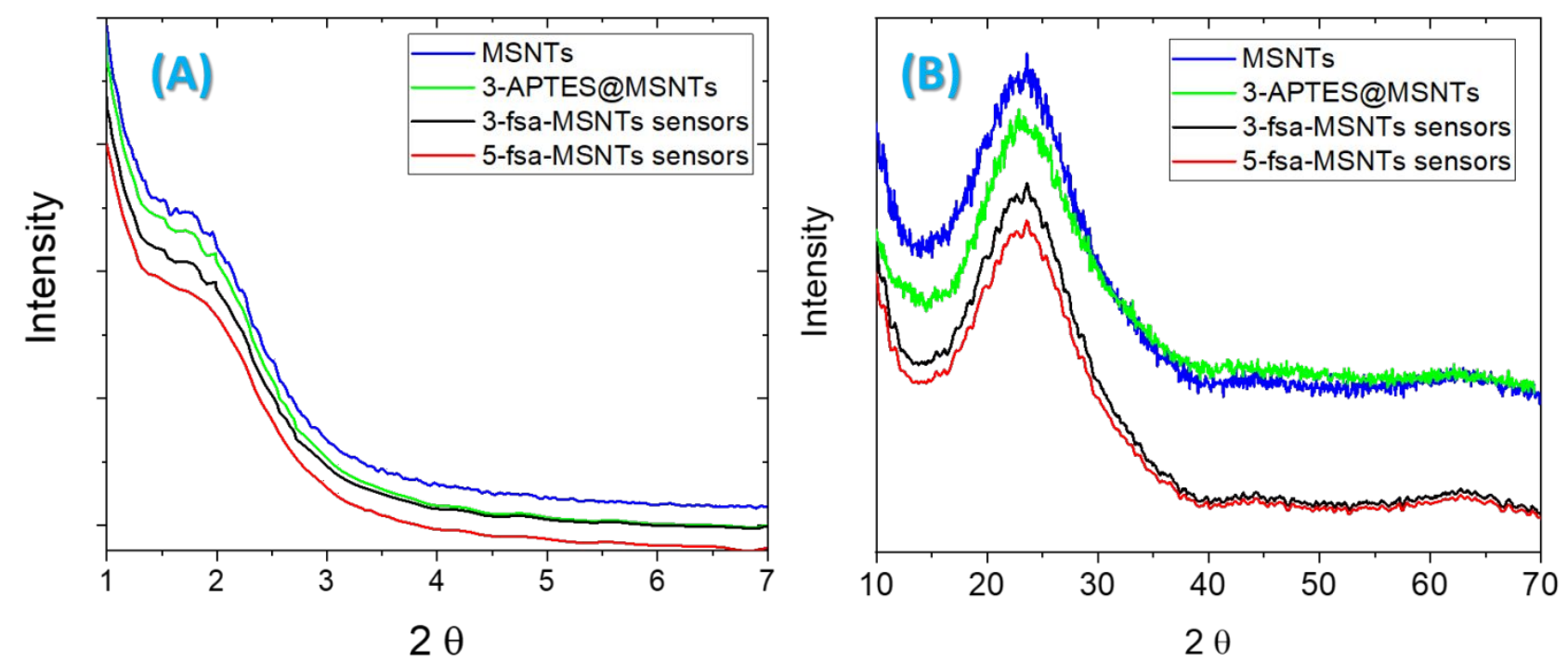

Figure S2. (A) Low-angle XRD patterns and (B) Wide-angle X-ray diffraction patterns of the MSNTs, 3-APTES@MSNTs, 3-fsa-MSNTs and 5-fsa-MSNTs sensors samples. 


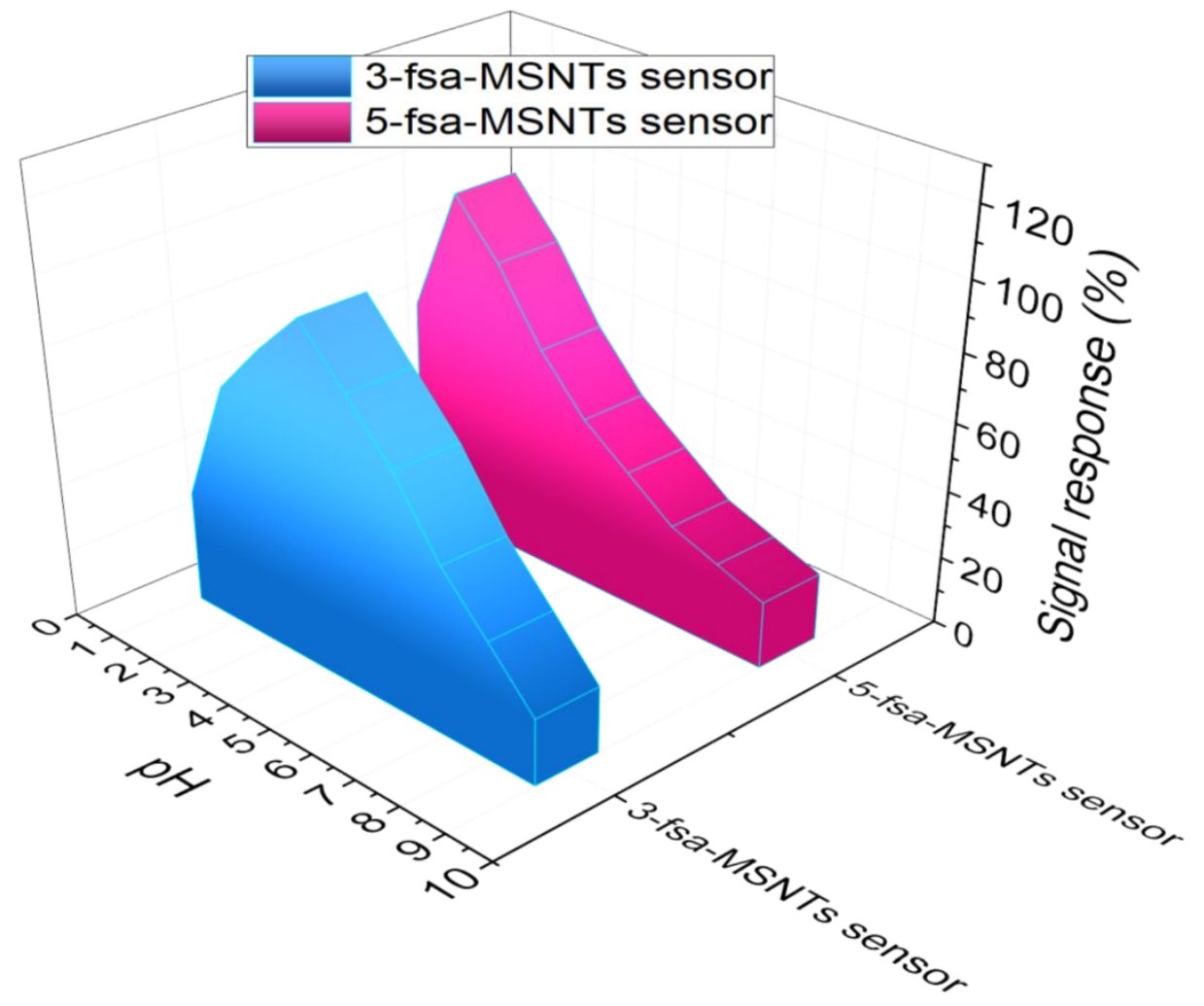

Figure S3. The effect of $\mathrm{pH}$ on the complexation of the Fe(III) with 3-fsa-MSNTs and 5-fsaMSNTs sensors at the maximum wavelengths of 378 and $495 \mathrm{~nm}$, respectively. 


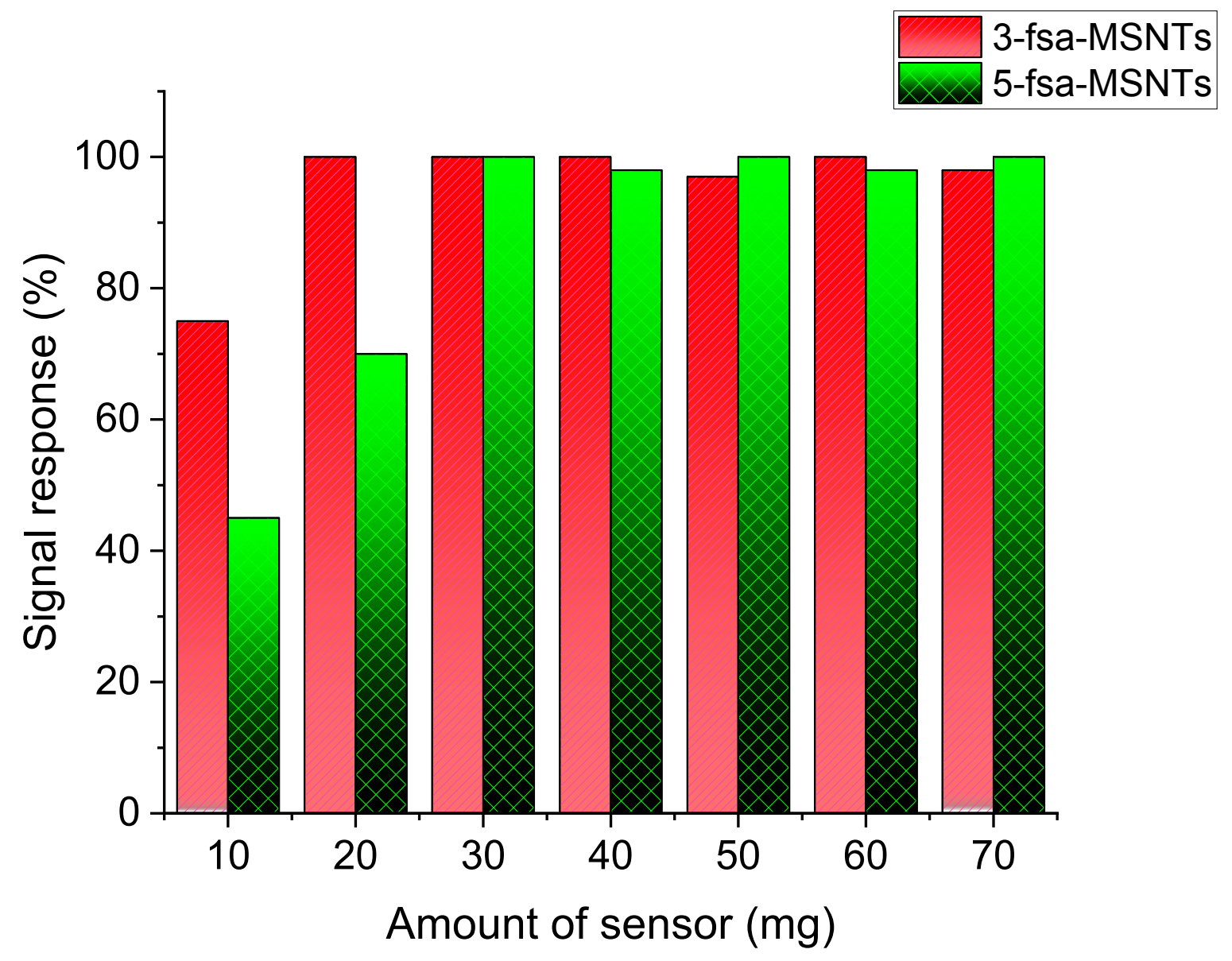

Figure S4. The effect of the amount of 3-fsa-MSNTs and 5-fsa-MSNTs sensors with $0.1 \mathrm{ppm}$ $\mathrm{Fe}(\mathrm{III})$ ions on the signal response at the wavelengths of 378 and $495 \mathrm{~nm}$, respectively. 


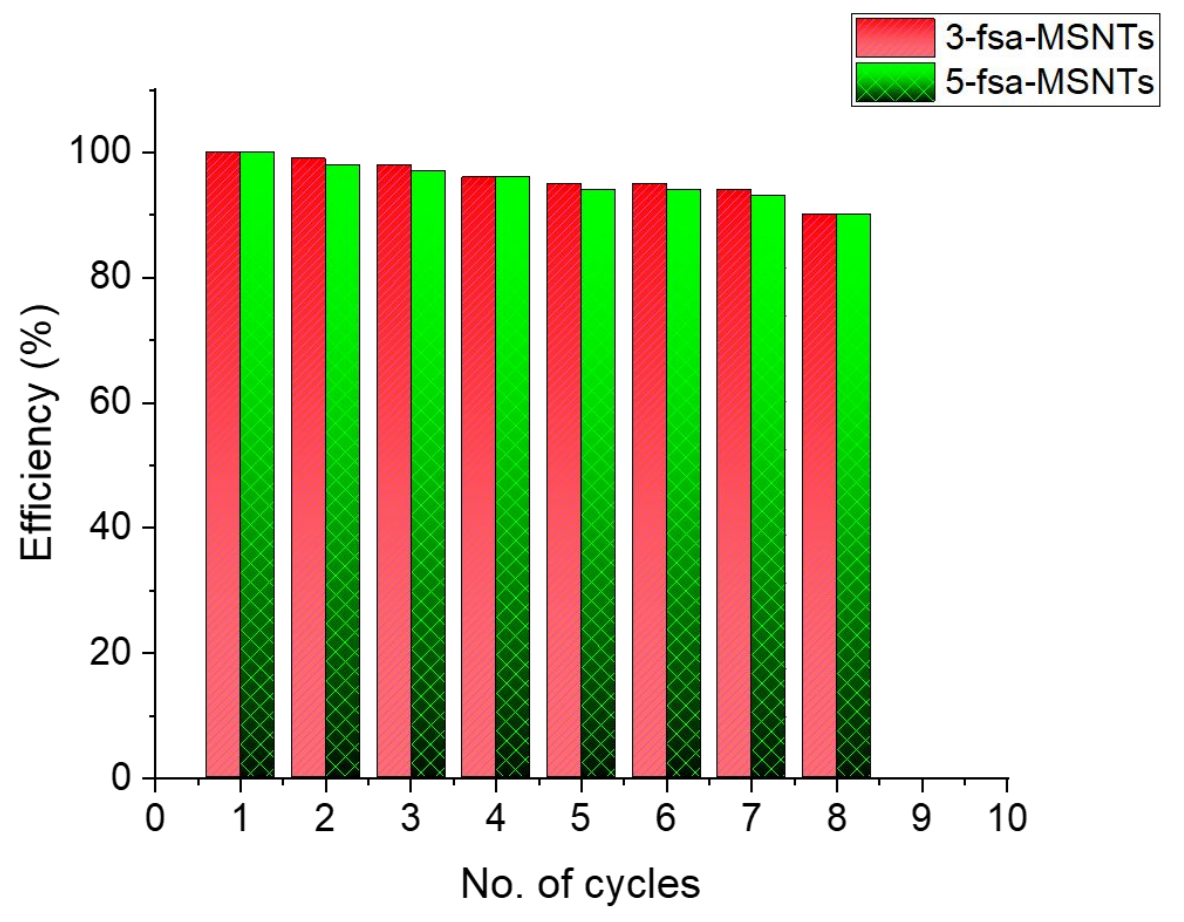

Figure S5. Reusability study of the 3-fsa-MSNTs and 5-fsa-MSNTs sensors in several cycles of sensing operations. 


\section{References}

(1) Abou-Melha, K. S.; Al-Hazmi, G. A. A.; Habeebullah, T. M.; Althagafi, I.; Othman, A.; ElMetwaly, N. M.; Shaaban, F.; Shahat, A. Functionalized silica nanotubes with azo-chromophore for enhanced Pd2+ and Co2+ ions monitoring in E-wastes, J. Mole. Liq. 3292021115585.

(2) Altalhi, T. A.; Ibrahim, M. M.; Mersal, G. A. M.; Alsawat, M.; Mahmoud, M. H. H.; Kumeria, T.; Shahat, A.; El-Bindary, M. A. Mesopores silica nanotubes-based sensors for the highly selective and rapid detection of $\mathrm{Fe} 2+$ ions in wastewater, boiler system units and biological samples. Anal. Chim. Acta 20211180338860. 